DOI: http://dx.doi.org/10.18524/1810-4215.2018.31.144461

\title{
ON THE CONFIGURATION SPACE OF A SPHERICALLY SYMMETRIC SYSTEM OF GRAVITATIONAL AND ELECTROMAGNETIC FIELDS
}

\author{
V.D. Gladush \\ Oles Honchar Dnipro National University, Dnipro, Ukraine, \\ vgladush@gmail.com
}

\begin{abstract}
We study some classical and quantum aspects of the configuration space for a sphericallysymmetric (SS) system of gravitational and electromagnetic fields. Note that this fields configurations, which are stationary respect to external observer, have regions of space-time (S-T) with dynamic behavior. This means that in these regions there exists an evolution of the S-T geometry in time, which is responsible for both classical and quantum mechanical properties of the model. From the standard action, we construct the reduced action and conserved total mass and charge. In view of a Hamiltonian constraint the non-dynamic degree of freedom from the action is excluded. This leads to the action in the minisuperspace. Therefore, the classical investigation stage of the Einstein equations solutions reduces to the study of solutions of the Einstein-Hamilton-Jacobi equation in the minisuperspace. It turns out that minisuperspace is flat therefore solutions of the Einstein equations correspond to a pencil of lines in the minisuperspace. Their intersections with the light cone of the minisuperspace correspond to the event horizons in the S-T of the charged $\mathrm{BH}$. The consideration of the quantum aspects is formally reduced to the quantization of a particle in a three-dimensional pseudo-Euclidean space. Using the compatibility condition of the DeWitt and the eigenvalue equations for the operators of mass and charge the configuration wave function is constructed. Thus, we obtain a model of a charged BH with a continuous spectrum of masses and charge.
\end{abstract}

Keywords: spherical-symmetric configurations, minisuperspace, Hamilton operator, mass and charge operators, compatibility condition

АБСТРАКТ. Досліджуються деякі класичні i квантові аспекти фізики i геометрії мінісуперпростору сферично-симетричної системи гравітаційного та електромагнітного полів. Відзначимо, що такі класичні конфігурації, які $€$ стаціонарними 3 точки зору зовнішнього спостерігача, мають певні області простору-часу (ПЧ) 3 динамічним поведінкою (Т-області). Це означає, що в цих областях існує еволюція геометрії ПЧ в часі, яка відповідає, як за класичні, так і за квантово-механічні властивості моделі. Зі стандартної дії будується дія для зазначеної системи полів в Т-області, вводяться повна маса і заряд системи, які зберігаються. За допомогу гамільтонової в'язі, з отриманої скороченої дії виключається нединамічна ступінь вільності. Це призводить до дії в конфігураційному просторі (мінісуперпросторі). Виявляється, що рівняння геодезичних в мінісуперпросторі разом 3 в'яззю еквівалентні рівнянням Ейнштейна. Тому, класичний етап дослідження рішення рівнянь Ейнштейна зводиться до дослідження розв'язків рівняння Ейнштейна-ГамільтонаЯкобі в мінісуперпросторі. Виявляється, що мінісуперпростор $\epsilon$ плоским, тому розв'язкам рівнянь Ейнштейна відповідає пучок прямих в мінісуперпросторі. Їх перетин зі світловим конусом мінісуперпростору відповідають горизонтам подій в ПЧ зарядженої ЧД. Перетин цих прямих у пучку відповідає центральній сингулярності ПЧ. Розгляд квантових аспектів системи формально зводиться до квантування вільної частинки в тривимірному псевдоевклідовому мінісуперпросторі. Використовуючи умову сумісності рівнянь Девітта і проблеми на власні значення квантових операторів маси i заряду, будується хвильова функція сферично-симетричної конфігурації гравітаційного і електромагнітного полів. Таким чином, ми отримуємо модель зарядженої чорної діри із безперервним спектром мас і заряду.

Ключові слова: сферично-симетричні конфігурації, конфігураційний простір, оператор Гамільтона, оператори маси i заряду, умова сумісності 


\section{Introduction}

It is known that the classical and quantum aspects of the behavior of the gravitational field is determined by a superspace metric so that superspace is the action arena of classical and quantum geometrodynamics. By studying the superspace geometry we can obtain important information about the classical and quantum manifestations of the dynamical system under consideration. However, the study of superspace in the general case meets with insurmountable mathematical difficulties (Anderson 2015, Giulini 2009). Therefore, reduced models are widely used, among which spherically symmetric (SS) configurations are popular and simplest models used for studying the problems of quantum gravity in a simpler setting. The general geometrodynamics approach to studying of the SS gravitational field of the black hole $(\mathrm{BH})$ was developed in work of Kuchař 1994, the case of the electromagnetic and gravitational fields configuration of the charged BH, was considered by Louko et al. 1996 and Nakamura et al. 1993.

The present work is devoted to studying of the minisuperspace of the electromagnetic and gravitational fields SS configurations and the search for a correspondence between space-time and the minisuperspace, with the subsequent transition to quantization. We consider the class of SS configurations with diagonal S$\mathrm{T}$ metrics. The model is based on the observation that the classical SS configurations of the electromagnetic and gravitational fields, which are stationary from the point of view of an external observer, have certain $\mathrm{S}-\mathrm{T}$ regions (T-regions) with dynamic behavior. This means that in these regions there is an evolution of the S-T geometry over time, which is responsible for the quantum mechanical properties of the considered charged $\mathrm{BH}$ model.

2. Classical description of the sphericallysymmetric configuration of the gravitational and electromagnetic fields

Consider the SS space-time $M^{(4)}$ with the metric

$$
d s^{2}=g_{\mu \nu} d x^{\mu} d x^{\nu}=\gamma_{a b} d x^{a} d x^{b}-R^{2} d \sigma^{2} .
$$

Here $d \sigma^{2}=d \theta^{2}+\sin ^{2} \theta d \alpha^{2}, R=R\left(x^{a}\right), \gamma_{a b}=$ $\gamma_{a b}\left(x^{a}\right)-2 \mathrm{D}$ metric tensor, $\sqrt{-g}=\sqrt{-\gamma} R^{2} \sin \theta$, где $g=\operatorname{det}\left|g_{\mu \nu}\right|, \gamma=\operatorname{det}\left|\gamma_{a b}\right|, \mu, \nu=0,1,2,3 ; a, b=0,1$. The action for a system of gravitational and electromagnetic fields has the form

$$
S=-\frac{1}{16 \pi c} \int\left(\frac{c^{4}}{\kappa}{ }^{(4)} R+F^{\mu \nu} F_{\mu \nu}\right) \sqrt{-g} d^{4} x,
$$

where ${ }^{\left.{ }^{4}\right)} R$ is the scalar curvature, $F_{\mu \nu}=A_{\nu, \mu}-A_{\mu, \nu}$ is the electromagnetic field tensor, $A_{\mu}=\left\{A_{a}, 0,0\right\}$ is vector potential.
Note that information about the structure of SS space is contained in the square of the gradient (see Berezin 2003)

$$
(\nabla R)^{2}=\gamma^{a b} R_{, a} R_{, b} .
$$

The surfaces $R\left(r, x^{0}\right)=$ const, for which $(\nabla R)^{2}=0$, divide $M^{(4)}$ into

$$
\begin{aligned}
& \text { R-regions } M_{R}^{(4)} \subset M^{(4)}, \text { when }\left((\nabla R)^{2}<0\right. \text { and } \\
& \text { T-regions } M_{T}^{(4)} \subset M^{(4)} \text {, when }\left((\nabla R)^{2}>0 .\right.
\end{aligned}
$$

In the R-region the surface $R\left(r, x^{0}\right)=$ const are timelike, and in the T-region is spacelike. Using the generalized Birkhoff theorem, in the R-region we can choose a coordinate system in which $\gamma_{a b}$ and $R$ depend only on the space-like coordinate $r$. Similarly, in the T-region, there exists an system in which $\gamma_{a b}$ and $R$ depend on the time-like coordinate $x^{0}$.

In work of Gladush (2017) it is shown that the metric (1) and action (2) in the T-region can be presented in the form

$$
\begin{gathered}
d s_{T}^{2}=h^{-1}\left(N d x^{0}\right)^{2}-h\left(d x^{1}\right)^{2}-R^{2} d \sigma^{2} \\
=R \xi^{-1}\left(N d x^{0}\right)^{2}-\xi R^{-1}\left(d x^{1}\right)^{2}-R^{2} d \sigma^{2} . \\
S_{T}=\int L_{T} d x^{0}=\int \frac{l}{2 c}\left\{\frac{\mathfrak{T}}{N}+\frac{c^{4}}{\kappa} N\right\} d x^{0},
\end{gathered}
$$

where $L_{T}$ is the Lagrange function of the reduced system with a kinetic term

$$
\mathfrak{T}=-\frac{c^{4}}{\kappa} \xi_{, 0} R_{, 0}+R^{2} \phi_{, 0}^{2} .
$$

Here $R_{, 0}=\partial R / \partial x^{0}, \xi_{, 0}=\partial \xi / \partial x^{0}$. In the R-region, the action $S_{R}$ has a similar form, the evolutionary coordinate $x^{0}$ is space-like here.

From the Lagrangian $L_{T}$ the primary constraint $P_{N}=\partial L / \partial \dot{N}=0$ and momenta $P_{i}=\partial L / \partial \dot{q}^{i}$ follow:

$P_{\xi}=-\frac{c^{3} l}{2 \kappa N} \dot{R}, \quad P_{R}=-\frac{c^{3} l}{2 \kappa N} \dot{\xi}, \quad P_{\phi}=\frac{l}{c N} R^{2} \dot{\phi}$.

From the Lagrange-Euler equation we obtain the secondary constraint

$$
\frac{\delta L_{T}}{\delta N}=\frac{\partial L_{T}}{\partial N}=\frac{l}{2 c}\left\{-\frac{\mathfrak{T}}{N^{2}}+\frac{c^{4}}{\kappa}\right\}=0 .
$$

So the Hamiltonian function $H=P_{\xi} \dot{\xi}+P_{R} \dot{R}+P_{\phi} \dot{\phi}-L$ leads to the Hamiltonian constraint in the T-region

$$
H=\frac{N c}{2 l}\left\{-\frac{4 \kappa}{c^{4}} P_{\xi} P_{R}+\frac{1}{R^{2}} P_{\phi}^{2}-\mu^{2}\right\} \sim 0,
$$

where $\mu=c l / \sqrt{\kappa}$.

In addition the system has the following conserved values: 
Charge function is equal to the charge inside the region of radius $\mathrm{R}$

$$
Q\left(N, R, \phi_{, 0}\right)=\frac{R^{2}}{N} \phi_{, 0}=\frac{c}{l} P_{\phi}
$$

Total mass function taking into account the contribution of the electromagnetic field is (see Gladush 2017)

$$
M_{t o t}=\frac{c^{2}}{2 \kappa} R\left(1+\gamma^{a b} R_{, a} R_{, b}\right)+\frac{Q^{2}}{2 c^{2} R},
$$

In the considered variables, as well as through momenta, it has the form

$$
\begin{gathered}
M_{t o t}=\frac{c^{2}}{2 \kappa}\left[R+\frac{1}{N^{2}}\left(\xi \dot{R}^{2}+\frac{\kappa R^{3} \dot{\phi}^{2}}{c^{4}}\right)\right], \\
M_{t o t}=\frac{1}{2 l^{2}}\left[\frac{l^{2} c^{2}}{\kappa} R+\frac{4 \kappa}{c^{4}} \xi P_{\xi}^{2}+\frac{1}{R} P_{\phi}^{2}\right] .
\end{gathered}
$$

We also write out the Poisson brackets of dynamic quantities:

$$
\left\{H, M_{t o t}\right\}=\frac{2 \kappa}{l^{2} c^{4}} P_{\xi} H \sim 0, \quad\{H, Q\}=\left\{M_{t o t}, Q\right\}=0 .
$$

3. The configuration space of the SS system of gravitational and electromagnet fields and its geometry

The factor $\mathrm{N}$ can be excluded from the action (5), while the original variational principle is transformed into a variational principle in the configuration space. Indeed, (8) implies $N=\sqrt{\kappa \mathfrak{T}} / c^{2}$. Substituting this expression into (5) we get

$$
S_{\mathfrak{R}}=\int L_{\mathfrak{R}} d x^{0}=\mu \int \sqrt{\mathfrak{T}} d x^{0}=\mu \int d \Omega,
$$

where

$$
d \Omega^{2}=\mathfrak{T}\left(d x^{0}\right)^{2}=-\frac{c^{4}}{\kappa} d \xi d R+R^{2} d \phi^{2}>0
$$

is the metric minisuperspace $\mathfrak{R}$. We see that $S_{\mathfrak{R}}$ is the action for a geodesic in the configuration space. The geodesic equations derived from this, together with the equation for $\mathrm{N}$, are equivalent to the original Einstein equations. Definitions of the momenta (7) can now be rewritten in the standard form

$$
P_{a}=\mu \Omega_{a b} \frac{d q^{a}}{d \Omega}
$$

as momenta of the particle with mass $\mu$ and 3-velocity $d q^{a} / d \Omega=\{d \xi / d \Omega, d R / d \Omega, d \phi / d \Omega\}$, moving along the geodesic in a minisuperspace.
It turns out that the minisuperspace with the metric (15) is flat. Therefore, there are transformations of field functions, for example,

$$
\begin{array}{r}
\xi=\frac{\kappa}{c^{4}}\left(c \tau-x-\frac{y^{2}}{c \tau+x}\right), \\
\phi=\frac{y}{c \tau+x}, R=c \tau+x
\end{array}
$$

leading metrics (15) to Lorentz form

$$
d \Omega^{2}=-c^{2} d \tau^{2}+d x^{2}+d y^{2} .
$$

Substituting $P_{\xi}=\partial S / \partial \xi, P_{R}=\partial S / \partial R, P_{\phi}=\partial S / \partial \phi$ into the Hamiltonian constraint (9), we arrive at the Einstein-Hamilton-Jacobi (EHJ) equation

$$
-\frac{4 \kappa}{c^{4}} \frac{\partial S}{\partial \xi} \frac{\partial S}{\partial R}+\frac{1}{R^{2}}\left(\frac{\partial S}{\partial \phi}\right)^{2}=\frac{l^{2} c^{2}}{\kappa} .
$$

His solution is

$$
S=\frac{l}{c} Q \phi+P_{\xi} \xi-\frac{l^{2} c^{6}}{4 \kappa^{2} P_{\xi}}\left(\frac{\kappa Q^{2}}{c^{4} R}+R\right) .
$$

We define the BH mass by the equation $M_{t o t}=m$. Then

$$
P_{\xi}= \pm l c^{2} \sqrt{\frac{m}{2 \kappa \xi_{0}}} .
$$

As a result, the trajectories equations in the minisuperspace take the form

$$
\phi(T)=\sqrt{\frac{\xi_{0}}{2 \kappa m}} \frac{Q}{T}, \xi(T)=\frac{c^{3}}{2 \kappa m} \xi_{0} T F_{T}(T, m, Q),
$$

where in the T-region $R=c T$ and

$$
F_{T}(T, m, Q)=-1+\frac{2 \kappa m}{c^{3} T}-\frac{\kappa Q^{2}}{c^{6} R^{2}}
$$

For the metric function in (4) we find

$$
h=\frac{c^{2} \xi_{0}}{2 \kappa m} F_{T}(T, m, q), h=-\frac{c^{2} \xi_{0}}{2 \kappa m} F_{R}(R, m, q)
$$

for the $\mathrm{T}$ - and R-regions, respectively, and $F_{R}(R, m, q)=-F_{T}(T=R / c, m, q)$. These expressions lead to standard representations of the Reissner-Nordstrom metric. in the T- and R-regions.

Consider the trajectories structure in the configuration space. The region of admissible motions corresponding to a solution in a T-region is determined by the conditions $\xi>0, R>0$ or

$$
-\Omega^{2}=c^{2} \tau^{2}-x^{2}-y^{2}>0, \quad R=c \tau+x>0,
$$

which correspond to the upper interior of the cone $\Omega^{2}=0$ in coordinates $\{c \tau, x, y\}$ (see fig. 1). In these 


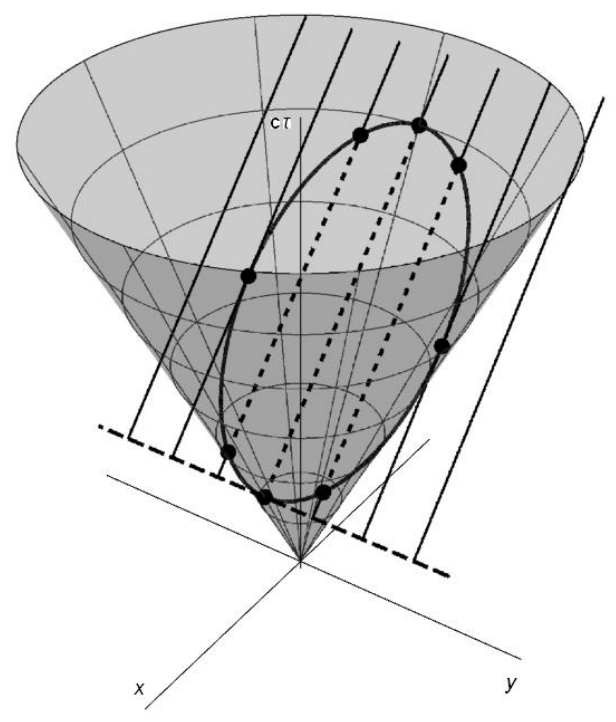

Figure 1: Mini-superspace SS configuration of gravitational and electromagnetic fields in coordinates $\{c \tau, x, y\}$.

coordinates, solutions (22) are described by straight lines:

$$
\begin{gathered}
c \tau(R)=\frac{1}{2}\left[\left(1-\frac{a}{R_{g}}\right) R+a\right], \\
x(R)=\frac{1}{2}\left[\left(1+\frac{a}{R_{g}}\right) R-a\right], \\
y=\sqrt{\frac{\xi_{0}}{R_{g}}} Q, \quad R_{g}=\frac{2 \kappa m}{c^{2}}, \quad a=\frac{\xi_{0} c^{4}}{\kappa}
\end{gathered}
$$

The evolution of the system in configuration space, for given $M$ and $Q$, can be represented as motion along a straight of the family (26), starting outside the cone $\Omega^{2}=0$ on the line $c \tau(0)=a / 2, x(0)=-a / 2, y=\sqrt{\xi_{0} / R_{g}} Q$, (central singularity). Further, the straight intersects the cone $\Omega^{2}=0$ with $R=R_{1}$ (inner horizon), comes upon inside the cone, crosses it with $R=R_{2}$ (outer horizon) and leaves to infinity of the minisuperspace. The motion inside the cone $\Omega^{2}=0$ of the minisuperspace corresponds to the solution for the T-region of the $\mathrm{S}-\mathrm{T}$, to the motion outside the cone corresponds to the solution for the R-regions of the S-T (see Fig. 1). For an extremely charged $\mathrm{BH}$, when $|Q|=m \sqrt{\kappa}$, the corresponding straight of the family (26) touches the cone $\Omega^{2}=0$ at the point $R_{1}=R_{2}=m \kappa$. For superextremal charges $|Q|>m \sqrt{\kappa}$ these straights lie outside the cone $\Omega^{2}=0$.

4. Quantization of a spherically symmetric configuration of the gravitational and electromagnetic fields
The quantum states of the field configuration under consideration are determined by the wave function $\Psi(R, \xi, \phi)$ on the minisuperspace with the coordinates $\{R, \xi, \phi\}$. The corresponding momentum operators in this representation have the form:

$$
\hat{P}_{R}=-i \hbar \frac{\partial}{\partial R}, \quad \hat{P}_{\xi}=-i \hbar \frac{\partial}{\partial \xi}, \quad \hat{P}_{\phi}=-i \hbar \frac{\partial}{\partial \phi} .
$$

The classical Hamiltonian, the total mass and charge functions lead to operators

$$
\begin{gathered}
\hat{H}=\frac{N c}{2 l}\left\{\frac{4 \kappa \hbar^{2}}{c^{4}} \frac{\partial^{2}}{\partial R \partial \xi}-\frac{\hbar^{2}}{R^{2}} \frac{\partial^{2}}{\partial \phi^{2}}-\frac{c^{2} l^{2}}{\kappa}\right\}, \\
\hat{M}=\frac{1}{2 l^{2}}\left(\frac{l^{2} c^{2}}{\kappa} R-\frac{4 \kappa \hbar^{2}}{c^{4}} \frac{\partial}{\partial \xi} \xi \frac{\partial}{\partial \xi}-\frac{\hbar^{2}}{R} \frac{\partial^{2}}{\partial \phi^{2}}\right) . \\
\hat{Q}=\frac{c}{l} \hat{P}_{\phi}=-i \frac{c \hbar}{l} \frac{\partial}{\partial \phi} .
\end{gathered}
$$

For the Hermitian operator of the total mass, in the configuration space we use the following ordering of the operators: $\hat{P}_{\xi} \xi \hat{P}_{\xi}$. The following commutation relations hold

$$
[\hat{H}, \hat{M}]=-\frac{2 \kappa \hbar^{2}}{l^{2} c^{4}} \frac{\partial}{\partial \xi} \hat{H} \sim 0, \quad[\hat{H}, \hat{Q}]=[\hat{Q}, \hat{M}]=0 .
$$

States with a certain total mass and charge correspond to eigenfunctions and eigenvalues of the operators of total mass and charge:

$$
\hat{M} \Psi_{m}=m \Psi_{m}, \quad \hat{Q} \Psi_{q}=q \Psi_{q} .
$$

They reduce to the following equations

$$
\begin{gathered}
\left\{\frac{c^{2} l^{2}}{\kappa} R-\frac{4 \kappa \hbar^{2}}{c^{4}} \frac{\partial}{\partial \xi} \xi \frac{\partial}{\partial \xi}-\frac{\hbar^{2}}{R} \frac{\partial^{2}}{\partial \phi^{2}}\right\} \Psi_{m}=2 l^{2} m \Psi_{m} \\
\frac{\partial}{\partial \phi} \Psi_{q}=\frac{i q l}{c \hbar} \Psi_{q}
\end{gathered}
$$

From the last equation we obtain $\Psi_{q}=A e^{i(q l / c \hbar) \phi}$ The general wave functions of the DeWitt equation $\hat{H} \Psi=0$ and the charge operator, also as general wave functions of the operators total mass and charge, can be represented in the form

$$
\Psi=\psi(\xi, R) e^{i(q l / c \hbar) \phi}, \quad \Psi_{m}=\psi_{m}(\xi, R) e^{i(q l / c \hbar) \phi} .
$$

The functions $\psi$ and $\psi_{m}$ satisfy the equations

$$
\begin{gathered}
\left(\frac{4 \partial^{2}}{\partial R \partial \xi}+\frac{c^{2} q^{2} l^{2}}{\kappa \hbar^{2}} \frac{1}{R^{2}}\right) \psi=\frac{c^{6} l^{2}}{\kappa^{2} \hbar^{2}} \psi \\
\left\{\frac{l^{2} c^{2}}{\kappa} R-\frac{4 \kappa \hbar^{2}}{c^{4}} \frac{\partial}{\partial \xi} \xi \frac{\partial}{\partial \xi}+\frac{1}{R} \frac{q^{2} l^{2}}{c^{2}}\right\} \psi_{m}=2 m l^{2} \psi_{m} .
\end{gathered}
$$

Using the compatibility condition for the DeWitt and eigenvalues equations for the mass operator, we construct a regular solution of this system on the horizon, 
which leads to a wave function of a configuration in a state with given mass $m$ and charge $q$ for a T-region:

$$
\Psi_{m, q}^{T}=C J_{0}\left(\frac{l c}{l_{p l}^{2}} T \sqrt{h F_{T}(T, m, q)}\right) e^{i(q l / c \hbar) \phi},
$$

where $J_{0}$ is the Bessel function of the first kind of zero order. The functions $F_{T}(T, m, q)>0$ is defined in (23). Note that $h$ and $T$ are positive independent values here. For the classical Reissner-Nordstrom solution, the variables $h$ and $R=c T$ enter the initial metric (4), at that in the T-region, the value of $h$ depends on $T$, according to $(24)$. We note that for the metric mini-superspace (15), signature conditions are not violated in both the $\mathrm{T}$ - and R-regions of the S-T. At that the evolution of the system in configuration space in all cases occurs within the direction $d \Omega^{2}>0$. Therefore quantum equations constructed in a minisuperspace are performed independently of the type of the region of the $\mathrm{S}-\mathrm{T}$. Thus, the wave function of the system in the $\mathrm{R}-$ region has the form similar (34) and can be written out by formally replacing $h F_{T}(T, m, q) \rightarrow-h F_{R}(R, m, q)$ :

$$
\Psi_{m, q}^{R}=C J_{0}\left(\frac{l}{l_{p l}^{2}} R \sqrt{-h F_{R}(R, m, q)}\right) e^{i(q l / c \hbar) \phi} .
$$

Here $R>0$ and $h<0$. Since $F_{R}(R, m, q)>0$, then the value under the radical is positive. In the classical case, the value of $h$ is determined by the value of $R$ through the function $F_{R}(R, m, q)$, according to (24). The wave function (35) can be formally obtained based on the metric (4) and the Lagrangian of the configuration $L_{R}$ in the R-region, where the evolutionary parameter $x^{0}$ is spacelike. Therefore, the solution (35) can be considered an analytic continuation of the solution (34) through the horizons $F_{R}(R, m, q)=F_{T}(T, m, q)=0$. Note that the coefficient $N$ is not included in the wave function $\Psi_{m, q}(h, T, \phi)$, which determines the probability amplitude of the configuration $\{h, T, \phi ; m, q\}$, that is, the points $\{h, T, \phi\}$ of a minisuperspace, for given observables $m, q$. The mass and charge spectra of the $\mathrm{BH}$ in this approach are continuous.

\section{Discussion and conclusions}

As it has been shown, the configuration space of the SS system of electromagnetic and gravitational fields is flat, therefore to solutions of the Einstein equations there correspond straight lines in minisuperspace with the metrics(18). This greatly simplifies the differentialgeometric structure of EHJ equations solutions. In addition, metric functions can be expressed in terms of the natural invariant parameter corresponding to the geodesic in the minisuperspace. As a result, classical solutions for S-T metrics can be constructed without fixing the calibration, for an arbitrary lapse function.
We note also that the used differential equations determines only the local structure of the space, while the global structure needs to be redefine. Thus, the question of the mass spectrum is not solved at local approach, since the nature of the spectrum depends on the global properties of the geometry of the S-T and superspace, their structure as a whole, as well as the structure of the phase space of the system. Therefore, to obtain a discrete spectrum, additional differential-geometric and algebraic (group) structures are introduced on the space-time, configuration or phase spaces (see, for example, Barvinsky et al. 2001, Das et al. 2003 and references therein). As a rule, a discrete spectrum occurs in the presence of a potential well or compact geometry of the configuration or phase space leading to the finite motions.

\section{References}

Anderson E.: 2015, ArXiv:1412.0239v2, 29.

Giulini D.: 2009, Gen.Rel.Grav. 41, 785.

Kuchař K.: 1994, Phys. Rev. D50, 3961.

Louko J., Winters-Hilt S.: 1996, Phys. Rev. D54, 2647.

Nakamura K., Konno S., Oshiro Y., Tomimatsu A.: 1993, Prog. Theor. Phys. 90, 861.

Berezin B.A.: 2003, Физика элементарных частии, и атомного ядра $\mathbf{3 4}, 49$.

Gladush V.D.: 2017, Odessa Astr. Publ., 30, 19.

Barvinsky A., Das S., Kunstatter G.: 2001, Class. Quantum Grav., 18, 4845.

Das S., Ramadev P., Yajnik U. A., Sule A.: 2003, Phys. Lett. B565, 201. 